\title{
CLINICAL RESEARCH ARTICLE OPEN An educational intervention for NICU staff decreased maternal postpartum depression
}

\author{
Sari Ahlqvist-Björkroth ${ }^{1}$, Anna Axelin ${ }^{2}$, Riikka Korja ${ }^{1}$ and Liisa Lehtonen ${ }^{3}$
}

BACKGROUND: Mothers of preterm infants are at increased risk for postpartum depression, which may disturb parenting and child development. Strategies for prevention are needed. Therefore, we evaluated how an educational intervention for neonatal staff affected depression symptoms among mothers of preterm infants.

METHODS: The Close Collaboration with Parents intervention was implemented in the NICU at Turku University Hospital in Finland. Maternal depression was compared between the pre-intervention and post-intervention cohorts using the Edinburgh Postnatal Depression Scale. The eligible infants were born $\leq 1500 \mathrm{~g}$ without major anomalies and survived. Data were available from 145 and 93 mothers in the pre-intervention and post-intervention cohorts, respectively, at 4 and/or 6 months of corrected age.

RESULTS: The depression scores were significantly lower in the post-intervention cohort than in the pre-intervention cohort; the estimated difference was 2.54 points $(95 \% \mathrm{Cl}, 1.24-3.83), p<0.001$. A total of $10.3 \%$ of the mothers in the pre-intervention cohort and $2.1 \%$ in the post-intervention cohort exceeded the threshold for depression, $p=0.066$.

CONCLUSION: The Close Collaboration with Parents intervention decreased depression symptoms among the mothers of very preterm infants. Systematic educational intervention targeted to the whole NICU staff can potentially prevent postnatal depression among mothers of preterm infants.

Pediatric Research (2019) 85:982-986; https://doi.org/10.1038/s41390-019-0306-y

\section{INTRODUCTION}

Mothers of preterm infants are at an elevated risk for postpartum depression (PPD). The rates of mothers' PPD after preterm birth vary from 6 to $48 \% .^{1-4}$ This large variation is partly explained by differences in study populations, measurement points, screening methods, and cutoff points. Mothers' depressive symptoms are most prevalent during the early postpartum weeks and decrease throughout the first postpartum year. ${ }^{3}$ However, the proportion of mothers scoring above the cutoff of probable major depression has been shown to remain high (17-20\%) until the second year after preterm delivery.,5 Prolonged maternal PPD is associated with earlier gestational age, low birth weight, the infant's ongoing illness or disability, a lack of social support, and the accumulation of environmental risk factors. ${ }^{4}$

Maternal PPD can potentially disturb the early mother-infant relationship among term infants. Most often, the interaction of depressed mothers has been intrusive, controlling, and overstimulating or passive, withdrawn, and under-stimulating, and sometimes a fluctuation between these two styles. ${ }^{6,7}$ Similarly, it has been found that mothers of preterm infants with PPD lack positive affective involvement and communication, and they are passive in interactions with their infants. ${ }^{8}$ Early maternal PPD has also been associated with a full term child's insecure and disorganized attachment to his or her mother and subsequent problems in the child's development (e.g., lower IQ, language development challenges, internalizing and externalizing behavioral problems, and psychopathology). ${ }^{9-11}$ A recent Finnish study also found that maternal depressive symptoms are associated with behavioral and emotional problems among very low birth weight (VLBW) children. ${ }^{12}$

Evidence is emerging about methods to prevent maternal depression after neonatal intensive care. Four interventions (Creating Opportunities for Parent Empowerment [COPE], Family Nurture Intervention [FNI], Mother-Infant Transaction Program [MITP], and individualized family-based intervention), which were carried out primarily during hospitalization of the preterm infant, have been successful in decreasing maternal depressive symptoms at discharge and when the infant was 1,2 , and 4 months old. ${ }^{13-17}$ These interventions have some common components, such as supporting active involvement of the parents in infant care, supporting parenting and/or the parent-infant relationship, and providing emotional support for the parents. However, no single component has been identified as directly affecting PPD among mothers of preterm infants. ${ }^{18}$ In previous studies, these interventions have been targeted to individual parent-infant dyads, and participation has been restricted by several exclusion criterion, e.g., twins or multiple infants and single mothers. Studies are needed to evaluate whether the uptake of the core components of these interventions into routine NICU practice can decrease the prevalence of PPD among mothers of preterm infants.

Therefore, the aim of this study is to evaluate if PPD can be decreased by an intervention which educates the whole multiprofessional staff of a NICU to collaborate with parents and thereby, reaches all mothers and their infants in neonatal hospital care. Our hypothesis was that the Close Collaboration with Parents intervention, which facilitates staff ability to involve parents in

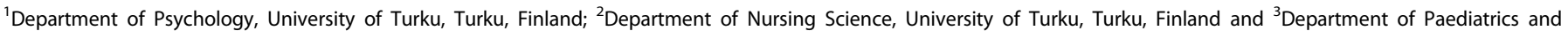
Adolescent Medicine, University of Turku and Turku University Hospital, Turku, Finland

Correspondence: Sari Ahlqvist-Björkroth (sarahl@utu.fi) 
Pre-intervention cohort

Years 2001-2006

Eligible and approached: Preterm infants born $\leq 1500 \mathrm{~g}$ and discharged alive and their mothers, $n=212$

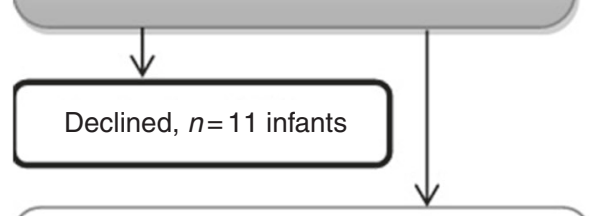

Participants:

Infants and their mothers, $n=201$

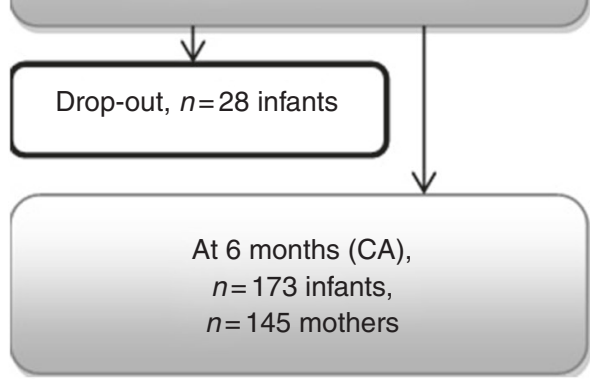

Fig. 1 Flow diagram of study participants

infant care, support parenting and the parent-infant relationship, and provide emotional support to parents, decreases depression among the mothers of very preterm infants.

\section{METHODS}

Participants

The study consisted of two cohorts from the same NICU. The preintervention cohort was recruited before the intervention from 2001 to 2006. The post-intervention cohort was recruited from 2011 to 2015. We included mothers (1) with infants with a birth weight of $\leq 1500 \mathrm{~g}$ who were alive at discharge without major congenital anomalies or syndromes, (2) who spoke Finnish or Swedish, and (3) who lived in the catchment area of the hospital. The pre-intervention cohort consisted of 145 of 212 eligible mothers of very low birth weight infants. These mothers belonged to the PIPARI follow-up study (The Development and Functioning of Very Low Birth Weight Infants from Infancy to School Age, www.utu.fi/pipari). The post-intervention cohort consisted of 93 of 173 eligible mothers of very low birth weight infants. The data of 55 mothers came for the Close Collaboration with Parents Intervention Study, ${ }^{19}$ and the data of 59 mothers came from clinical patient record data that were collected during their infants' 4-month follow-up visits (Fig. 1). Of these 114 mothers, the data were available for 21 mothers from both sources and, therefore, the total sample size of the post-intervention cohort was 93.

To ensure maximum recruitment, the same staff nurse approached all eligible mothers during both pre-intervention and post-intervention periods before discharge to home. After verbal and written information about the study, parents had 1 day to consider their participation. An informed consent was signed if the parents decided to participate in the study. Before discharge from the hospital, each mother received a folder with questionnaires for specific measurement time points. They filled out the PPD screener at home and returned it by mail to the research
Post-intervention cohort

Years 2011-2015

\section{Eligible:}

Preterm infants born $\leq 1500 \mathrm{~g}$ and

discharged alive and their mothers, $n=173$

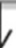

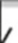

Drop-out, $n=6$ infants

Approached for the study or participated in clinical follow-up:

Infants and their mothers, $n=167$

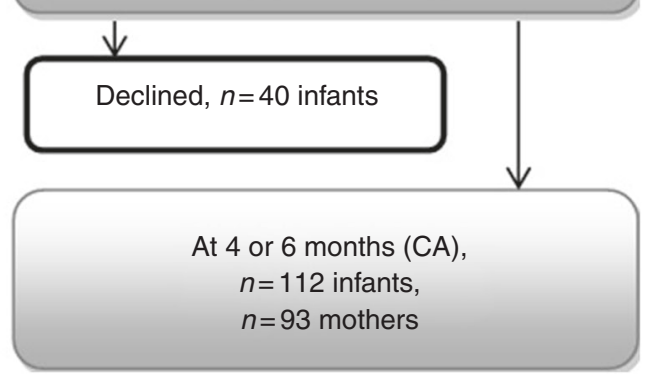

assistant. In the follow-up clinic, mothers filled out the PPD screener during their follow-up visits and returned it to a nurse or doctor.

Measures

PPD was assessed using the Edinburgh Postnatal Depression Scale (EPDS). ${ }^{20}$ The EPDS is a 10 -item, self-reported questionnaire for screening postpartum depression symptoms. The maximum score in the scale is 30 , and the minimum is 0 , with the higher scores indicating more depressive symptoms. In the analysis, the EPDS scores were used as continuous and categorical variables. A score of 13 or more was used to indicate probable major postpartum depression. $^{21}$

In the pre-intervention and the post-intervention cohorts, the mothers filled out the EPDS at the child's age of 6 months (CA). The mothers in the post-intervention cohort, whose EPDS scores were collected from the patient records, filled out the questionnaire at the child's age of 4 months (CA). The post-intervention cohort included 21 mothers who filled out the EPDS at the child's ages of 4 and 6 months. These mothers were approached with a separate written information and consent letter to request permission to compare their scores between the two age points. Those who agreed returned the signed consent form to a research assistant or agreed verbally via phone. In total, 21 agreements were received from the mothers.

\section{Intervention}

The intervention, Close Collaboration with Parents ${ }^{\mathrm{TM}}$, was carried out at the regional level III NICU in Finland from 2009 to 2012. The NICU had a traditional architecture with two to four patients per room and no additional sanitary or lounge facilities for the parents. There were 18 NICU beds. The intervention was a training program, which was targeted at the whole health care team of the unit, including medical and nursing staff members. The training of the whole team was carried out in two waves; the first half of the staff (28/51 nurses, 3/6 neonatologists) was trained between 2009 
and 2011 and the second half (21/51 nurses) between 2011 and 2012. The intervention and implementation has been described in greater detail elsewhere. $^{19}$

The goal of the training program was to increase the staff ability to (1) see and communicate the individual behaviors and needs of the infants, (2) actively listen to parents' perceptions of their babies' behaviors and collaboratively plan the care of the infants, (3) understand the individual features of families and create a basis for collaborative decision-making, and (4) integrate parents to care for their infants from admission through discharge and to plan discharge together.

The training program consisted of four phases, each built upon the previous one: (I) observing preterm infant behavior, (II) joint observation of babies with parents, (III) building understanding of individual characteristics of families, and (IV) family-centered transition from hospital to home. The intervention was planned to change values and attitudes, rather than single-care practices, to facilitate a transition in the care culture of the unit. Therefore, a core part of the training was to reflect on individual values and attitudes related to parenting support. The key elements of the training were (1) the involvement of the whole health care team, (2) the use of multiple learning/teaching modalities (theoretical education, bedside practicing, individual mentoring, and reflective supervision), (3) bedside practices with individual peer mentors, and (4) reflections on bedside practices in supervision groups led by a psychologist.

The unit carried out the training for the first half of the staff with extra resources allocated to the training in 2009 and 2010. The training continued for the second half of the staff after the first half was trained using a train-the-trainer method, which is still used to sustain the new care culture. Successful implementation was shown in a qualitative interview study among the staff. ${ }^{22}$

Ethics

The Close Collaboration with Parents Intervention Study was approved by the Ethics Committee of the Hospital District of Southwest Finland in February 2011, and the PIPARI study was approved by the same committee in December 2000. The hospital approved the review of the mothers' clinical patient records in June 2014.

\section{Statistical analyses}

The post-intervention cohort included 21 mothers who filled in the EPDS at both 4-month and 6-month time points. A sign test for paired data was used to compare their EPDS scores between these two time points. Thereafter, only the scores from the 6-month time point were used in the further analyses. Associations between background variables and EPDS scores were tested using an analysis of covariance. Mixed-model repeated measures analysis was used with the continuous EPDS mean score as response variables and background variables, with measurement time points and study cohorts used as the predictor variables. A subject was used as a random effect. The Wilcoxon-Mann-Whitney test was used to analyze whether the study groups were associated with EPDS scores above 12. No imputations were done for missing data.

Statistical analyses were conducted using SAS for Windows (version 9.4, SAS Institute, Cary, NC). $p$-values below 0.05 were considered statistically significant.

\section{Statistical measures}

We found no statistically significant differences between the EPDS scores of 4- and 6-month data in the post-intervention cohort ( $M$ $=4.25, \mathrm{SD}=3.85 ; \mathrm{M}=4.17, \mathrm{SD}=3.97$, respectively, $x^{2}=0.03, p=$ $0.86)$. We tested the significance of the following background factors: gestational weeks at birth, birth weight, sex, SGA status (defined as less than -2 SD or greater than or equal to -2 SD, according to the national reference values for gestational age, sex,

\begin{tabular}{|c|c|c|}
\hline Variables & $\begin{array}{l}\text { Pre-intervention } \\
(n=145)\end{array}$ & $\begin{array}{l}\text { Post intervention } \\
(n=93)\end{array}$ \\
\hline \multicolumn{3}{|l|}{ Infant } \\
\hline Male sex, No (\%) & $81(56)$ & $46(49)$ \\
\hline $\begin{array}{l}\text { Gestational age, } \\
\text { mean (SD), wk }\end{array}$ & $28.90(2.88)$ & $28.36(2.36)$ \\
\hline $\begin{array}{l}\text { Birth weight, mean } \\
\text { (SD), kg }\end{array}$ & $1077.04(288.93)$ & $1004.76(276.88)$ \\
\hline SGA (\%) & $53(39)$ & $27(29)$ \\
\hline LOS, median (SD), day & $60(38.04)$ & $64(45.50)$ \\
\hline \multicolumn{3}{|l|}{ Mother } \\
\hline Age, mean SD, yr & $30.98(5.13)$ & $31.29(5.71)$ \\
\hline Singleton, no. (\%) & $117(81)$ & $74(80)$ \\
\hline Vaginal birth, no. (\%) & $51(35)$ & $35(38)$ \\
\hline $\begin{array}{l}\text { Smoking during } \\
\text { pregnancy, no. (\%) }\end{array}$ & $24(17)$ & 8/64 (13) \\
\hline $\begin{array}{l}\text { Previous mental health } \\
\text { problems, no. (\%) }\end{array}$ & $18(13)$ & 10/55 (18) \\
\hline
\end{tabular}

parity, or multiple birth), the mode of delivery (vaginal or Csection), the total length of the initial hospital stay, multiple birth (singleton or multiple), older siblings (yes or no), smoking during pregnancy, and previous mental health diagnoses of the mother. Among background factors, only previous mental health problems of the mother were statistically significantly associated with the EPDS mean score, $\mathrm{F}=11.71, p<0.001$. However, the mental health history was available only in $60 \%$ of the mothers in the post-intervention cohort. The proportion of mothers with a history of mental health problems was the same for both the preintervention and post-intervention groups (Table 1). Birth weight was not included in the multivariate model because of its collinearity with gestational age.

\section{RESULTS}

The study included 145 mothers and their infants who belonged to the pre-intervention cohort and 93 mothers and their infants who belonged to the post-intervention cohort. The participant characteristics are summarized in Table 1.

The EPDS scores of the mothers in the post-intervention cohort were significantly lower than the EPDS scores of the mothers in the pre-intervention cohort $(M=4.32, S D=3.67 ; M=6.45, S D=$ 4.79 , respectively); the estimate of the difference was $2.54(95 \% \mathrm{Cl}$, from 1.24 to 3.83), $p<0.001$. The Cohen's $d=0.50$ implied a medium effect size. The effect of the intervention on maternal depression remained statistically significant when the analysis was adjusted by the background variables, $2.59(95 \% \mathrm{Cl}$, from 0.16 to 5.03), $p=0.04$. The proportion of mothers who scored above the EPDS cutoff was 15/145 (10.3\%) in the pre-intervention cohort and $2 / 93(2.1 \%)$ in the post-intervention cohort, $X^{2}=3.9, p=0.066$.

Furthermore, we used multivariate models to test the differences in EPDS scores based on each infant's gestational age at the time of birth. The results are illustrated in Fig. 2. The differences in EPDS scores between the pre-intervention and post-intervention cohorts were the greatest in the gestation week group from 30 to $31(M=6.31, S D=4.60 ; M=2.77, S D=3.37$, respectively), where the estimate of the difference was $4.22(95 \% \mathrm{Cl}$, from 0.56 to 7.89$)$ and $p=0.02$. The estimate of the difference was $3.64(95 \% \mathrm{Cl}$, from 1.03 to 6.25 ) among the mothers who delivered at 


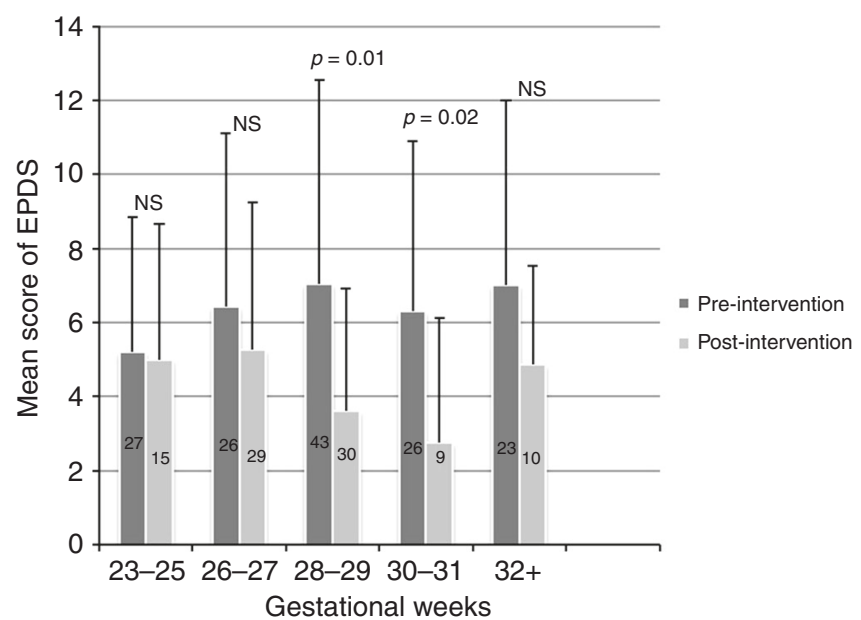

Fig. 2 Differences between pre-intervention and post-intervention cohorts in maternal EPDS scores according to infant's gestational age group. The number of mothers in each group is indicated by the number inside the bar

gestational weeks 28-29 $(M=7.05, S D=5.49 ; M=3.62, S D=$ 3.31 , respectively), $p=0.01$. In the other gestational age groups, the differences were not statistically significant. The estimate of the difference was $1.69(95 \% \mathrm{Cl}$, from -0.86 to 4.25$)$ in the gestation week group from 26 to $27(\mathrm{M}=6.42, \mathrm{SD}=4.71 ; \mathrm{M}=$ $5.03, \mathrm{SD}=4.00$, respectively), $p=0.19$, and in the group of mothers who delivered below 26 weeks of gestation, the estimate was $1.37(95 \% \mathrm{Cl}$, from -1.20 to 3.98$)$ (pre-intervention $\mathrm{M}=5.22$, $\mathrm{SD}=3.63$; post-intervention $\mathrm{M}=5.00, \mathrm{SD}=3.67$ ), $p=0.29$. The intervention effect was also not significant in the group of the most mature infants; the estimate was $2.66(95 \% \mathrm{Cl}$, from -33.48 to 38.81) (pre-intervention $\mathrm{M}=7.00, \mathrm{SD}=4.99$; post-intervention $\mathrm{M}=4.78, \mathrm{SD}=2.67), p=0.29$.

\section{DISCUSSION}

This study showed that the Close Collaboration with Parents intervention was effective in decreasing postpartum depression symptoms in mothers of very low birth weight infants. The postintervention mothers of preterm infants had comparable depression symptom scores to those reported in a low-risk population from the region. ${ }^{23}$ The prevalence rates of PPD in our study may seem low in comparison with many other studies. ${ }^{1,2,4}$ However, the prevalence rate in the pre-intervention cohort is comparable with those studies that had measured PPD 3 months postpartum or later and used rigorous screening cutoffs. ${ }^{3,24,25}$ The low rate of PPD in the pre-intervention cohort may also indicate that the unit already had a fairly high level of family-centered care before the intervention. We also found that long hospital care was not a risk factor for higher depression rates. Previous studies have reported mixed findings on the predictors of maternal PPD or distress. A recent review proposed that parents' psychological distress after preterm birth is in complicated ways related to, and mediated and moderated by intrapersonal, interpersonal, institutional, and public policy factors. ${ }^{26}$

The Close Collaboration with Parents intervention yielded the biggest effects in mothers who had given birth between 28 and 31 gestational weeks. We find it noteworthy that this salutogenic intervention seems to benefit a large number of very preterm infants as decreasing maternal depression will support later child development.

The study has some limitations. First, as the intervention was targeted to the whole unit staff, a randomized controlled study design was not applicable. As all NICU patients in the post- intervention period were exposed to the new care culture, all very preterm infants in the unit were eligible. ${ }^{19}$ The historical control group consisted of very preterm infants from the same unit and the same population. It is also important to note that the medical performance of the unit was stable during the time of data collection and intervention. The standardized neonatal mortality and morbidities were consistently at or below the average of a large peer-comparison network (Vermont Oxford Network), suggesting that there were no major changes in the medical care between the study cohorts and that the depression outcomes can be attributed to the intervention. Another limitation of the study was that recruitment was less successful in the post-intervention cohort compared to the pre-intervention cohort. This could reflect mothers' empowerment gained through the intervention. A third limitation to be considered is that the PPD was measured at 4-6 months of corrected age in the post-intervention cohort, compared to 6 months in the pre-intervention cohort. This wider time span was used to decrease attrition, thereby minimizing selection bias. We showed that there was no difference in the depression rate between these age points in those who replied at both time points.

Four controlled NICU interventions-COPE, ${ }_{16} \mathrm{FNI}^{15}{ }^{15} \mathrm{MITP},{ }^{13}$ and Individualized family-based intervention ${ }^{14}$ - have previously resulted in decreased PPD symptoms among preterm infants' mothers at the end of the hospital stay or when the infant was 1 , 2 , or 4 months old. Three of the four studies were randomized. ${ }^{13,15,16}$ Compared to these studies, ours had larger patient groups with the least selected patient populations. The previous studies used either the CES-D (Centre of Epidemiologic Studies Depression Scale) $^{27}$ or Beck Depression Inventory $(\mathrm{BDI}-\mathrm{II})^{28}$ to measure maternal postnatal depression. Although both methods are well known, valid, and reliable in measuring depressive symptoms in adults, they are not specifically developed to measure postnatal depressive symptoms, as the EPDS, used in this study, is.

The focus of the Close Collaboration with Parents intervention on supporting the parent-infant relationship may be the factor explaining decreased symptoms of maternal PPD. In addition to the Close Collaboration with Parents intervention, all four previous interventions shown to be effective in decreasing depression focused on supporting the parent-infant relationship. Some evidence shows that relationally focused treatments may be even more effective than traditional depression treatments in the treatment of maternal PPD. ${ }^{29,30}$ The Close Collaboration with Parents intervention and the Individualized Family-Based ${ }^{14}$ intervention also provided comprehensive support for the whole family. Similar to the Close Collaboration with Parents intervention, three ${ }^{14-16}$ out of the four interventions started soon after birth and lasted for the duration of the hospitalization. Therefore, the support for the parent-infant relationship was also long lasting in these interventions. The Close Collaboration with Parents intervention was unique in actively involving parents in the daily care of their infants from the beginning and in shared decision-making regarding the care planning.

Future research should confirm these findings in a randomized controlled study setting. Furthermore, studies are needed to evaluate the effects of a unit-wide intervention on later child outcomes and the ways in which mothers' better mental wellbeing mediates these effects. The essential and sufficient components in the training programs should be identified to make the programs more feasible, even with fewer resources. The Close Collaboration with Parents intervention has been implemented in 10 NICUs and thus has shown applicability in various NICU contexts.

The Close Collaboration with Parents intervention decreased symptoms of maternal postpartum depression among mothers of very preterm infants. Since it seems that providing the staff better 
skills to support parenting and the parent-infant relationship decreases the symptoms of maternal depression, NICUs with high rates of maternal depression should consider implementing interventions such as the Close Collaboration with Parents intervention. Maternal postpartum depression also seems to be a feasible and effective marker to monitor the quality of parent support in a NICU.

\section{ACKNOWLEDGEMENTS}

We thank Fulbright scholar, Ms Sarah Holdren, BA, for English language editing. The Paediatric Foundation and the South Western Finnish Fond of Neonatal Research have financially supported this study.

\section{AUTHOR CONTRIBUTIONS}

S.A.B. performed the literature search. S.A.B., A.A. and L.L. conceptualized the study and drafted the study protocol. S.A.B., A.A., R.K. and L.L. acquired, analyzed, and interpreted the data and planned the statistical analyses. S.A.B., A.A., R.K. and L.L. drafted and revised the manuscript. All authors had full access to the data in the study and take full responsibility for the integrity of the data and the accuracy of the data analysis. All authors have approved the final submitted manuscript and have agreed to be accountable for all aspects of the manuscript.

\section{ADDITIONAL INFORMATION}

Competing interests: The authors declare no competing interests.

Publisher's note: Springer Nature remains neutral with regard to jurisdictional claims in published maps and institutional affiliations.

\section{REFERENCES}

1. Helle, N. et al. Very low birth-weight as a risk factor for postpartum depression four to six weeks postbirth in mothers and fathers: cross-sectional results from a controlled multicentre cohort study. J. Affect Disord. 180, 154-161 (2015).

2. Lefkowitz, D. S., Baxt, C. \& Evans, J. R. Prevalence and correlates of posttraumatic stress and postpartum depression in parents of infants in the neonatal intensive care unit (NICU). J. Clin. Psychol. Med. Settings 17, 230-237 (2010).

3. Pace, C. C. et al. Evolution of depression and anxiety symptoms in parents of very preterm infants during the newborn period. JAMA Pediatr. 170, 863-870 (2016).

4. Vigod, S., Villegas, L., Dennis, C. \& Ross, L. Prevalence and risk factors for postpartum depression among women with preterm and low-birth-weight infants: a systematic review. BJOG: Int. J. Obstet. Gynaecol. 117, 540-550 (2010).

5. Huhtala, M. et al. Parental psychological well-being and cognitive development of very low birth weight infants at 2 years. Acta Paediatr. 100, 1555-1560 (2011).

6. Field, T. Postpartum depression effects on early interactions, parenting, and safety practices: A review. Infant. Behav. Dev. 33, 1-6 (2010).

7. Lovejoy, M. C., Graczyk, P. A., O'Hare, E. \& Neuman, G. Maternal depression and parenting behavior: a meta-analytic review. Clin. Psychol. Rev. 20, 561-592 (2000).

8. Korja, R. et al. Maternal depression is associated with mother-infant interaction in preterm infants. Acta Paediatr. 97, 724-730 (2008).

9. Goodman, S. H. et al. Maternal depression and child psychopathology: a metaanalytic review. Clin. Child Fam. Psychol. Rev. 14, 1-27 (2011).

10. Grace, S. L., Evindar, A. \& Stewart, D. E. The effect of postpartum depression on child cognitive development and behavior: a review and critical analysis of the literature. Arch. Women's. Ment. Health 6, 263-274 (2003).

11. Martins, C. \& Gaffan, E. A. Effects of early maternal depression on patterns of infant-mother attachment: a meta-analytic investigation. J. Child Psychol. Psychiatry 41, 737-746 (2000).

12. Huhtala, M. et al. Associations between parental psychological well-being and socio-emotional development in 5-year-old preterm children. Early Hum. Dev. 90, 119-124 (2014).

13. Ravn, I. H. et al. Effects of early mother-infant intervention on outcomes in mothers and moderately and late preterm infants at age 1 year: a randomized controlled trial. Infant. Behav. Dev. 35, 36-47 (2012).
14. Meyer, E. C. et al. Family-based intervention improves maternal psychological well-being and feeding interaction of preterm infants. Pediatrics 93, 241-246 (1994).

15. Welch, M. G. et al. Depression and anxiety symptoms of mothers of preterm infants are decreased at 4 months corrected age with family nurture intervention in the NICU. Arch. Women's. Ment. Health 19, 51-61 (2016).

16. Melnyk, B. M. et al. Improving cognitive development of low-birth-weight premature infants with the COPE program: a pilot study of the benefit of early NICU intervention with mothers. Res. Nurs. Health 24, 373-389 (2001)

17. Mendelson, T., Cluxton-Keller, F., Vullo, G. C., Tandon, S. D. \& Noazin, S. NICUbased interventions to reduce maternal depressive and anxiety symptoms: a meta-analysis. Pediatrics 139 https://doi.org/10.1542/peds.2016-1870 (2017).

18. Benzies, K. M., Magill-Evans, J. E., Hayden, K. A. \& Ballantyne, M. Key components of early intervention programs for preterm infants and their parents: a systematic review and meta-analysis. BMC Pregnancy Childbirth 13 (Suppl 1), S10 (2013).

19. Ahlqvist-Björkroth, S., Boukydis, Z., Axelin, A. M. \& Lehtonen, L. Close collaboration with parents ${ }^{\mathrm{TM}}$ intervention to improve parents' psychological well-being and child development: description of the intervention and study protocol. Behav. Brain. Res. 325, 303-310 (2017).

20. Cox, J. L., Holden, J. M. \& Sagovsky, R. Detection of postnatal depression. development of the 10 -item edinburgh postnatal depression scale. Br. J. Psychiatry 150, 782-786 (1987).

21. Matthey, S., Henshaw, C., Elliott, S. \& Barnett, B. Variability in use of cut-off scores and formats on the edinburgh postnatal depression scale: implications for clinical and research practice. Arch. Women's. Ment. Health 9, 309-315 (2006).

22. Axelin, A., Ahlqvist-Bjorkroth, S., Kauppila, W., Boukydis, Z. \& Lehtonen, L. Nurses' perspectives on the close collaboration with parents training program in the NICU. MCN Am. J. Matern. Child Nurs. 39, 260-268 (2014).

23. Ahlqvist-Bjorkroth, S. et al. Initiation and exclusivity of breastfeeding: association with mothers' and fathers' prenatal and postnatal depression and marital distress. Acta Obstet. Gynecol. Scand. 95, 396-404 (2016).

24. Agostini, F., Neri, E., Dellabartola, S., Biasini, A. \& Monti, F. Early interactive behaviours in preterm infants and their mothers: influences of maternal depressive symptomatology and neonatal birth weight. Infant. Behav. Dev. 37, 86-93 (2014).

25. Mörelius, E., Örtenstrand, A., Theodorsson, E. \& Frostell, A. A randomised trial of continuous skin-to-skin contact after preterm birth and the effects on salivary cortisol, parental stress, depression, and breastfeeding. Early Hum. Dev. 91, 63-70 (2015).

26. Loewenstein, K. Parent psychological distress in the neonatal intensive care unit within the context of the social ecological model: a scoping review. J. Am. Psychiatr. Nurses Assoc. 24, 495-509 (2018).

27. Radloff, L. The CES-D scale: a self-report depression scale for research in the general population. Appl. Psychol. Meas. 1, 385-401 (1977).

28. Beck, A., Steer, R. \& Brown, G. Beck Depression Inventory, 2nd edn Manual (Psychological Corporation, San Antonio, 1996).

29. Clark, R., Tluczek, A. \& Brown, R. A mother-infant therapy group model for postpartum depression. Infant Ment. Health J. 29, 514-536 (2008).

30. Forman, D. et al. Effective treatment for postpartum depression is not sufficient to improve the developing mother-child relationship. Dev. Psychopathol. 19, 585-602 (2007).

(c) (1) Open Access This article is licensed under a Creative Commons cc. Attribution-NonCommercial-ShareAlike 4.0 International License, which permits any non-commercial use, sharing, adaptation, distribution and reproduction in any medium or format, as long as you give appropriate credit to the original author(s) and the source, provide a link to the Creative Commons license, and indicate if changes were made. If you remix, transform, or build upon this article or a part thereof, you must distribute your contributions under the same license as the original. The images or other third party material in this article are included in the article's Creative Commons license, unless indicated otherwise in a credit line to the material. If material is not included in the article's Creative Commons license and your intended use is not permitted by statutory regulation or exceeds the permitted use, you will need to obtain permission directly from the copyright holder. To view a copy of this license, visit http://creativecommons.org/licenses/by-nc-sa/4.0/.

(c) The Author(s) 2019 